\title{
EXPANDING QUASARS AND THE EXPANSION OF THE UNIVERSE
}

\author{
M. H. Cohen, P. D. Barthel, T. J. Pearson, and J. A. Zensus \\ Owens Valley Radio Observatory \\ California Institute of Technology \\ Pasadena, California 91125 \\ USA
}

The $\mu-z$ diagram (Figure 1) plots the observed internal proper motion $\mu$ versus redshift $z$ for 32 extragalactic radio sources associated with active galactic nuclei. The observed points fall below an upper bound which decreases with redshift; there is a statistically significant anticorrelation between redshift and internal proper motion.

We only include sources for which measurements of internal motion are published (see the table of Zensus and Pearson in this volume). The selection is biased towards sources with large proper motions, which tend to be monitored intensively, but we are not aware of any selection biases which would introduce the observed correlation. The filled circles represent sources with well-established measurements, i.e., based on maps at three or more epochs where the components are well identified. The crosses represent sources with only limited observations or ill-defined proper motions.

The correlation between redshift and proper motion provides a powerful test of models of expanding sources and of theories of the origin of the redshift. We discuss here only the currently popular relativistic beam model, with standard Friedmann cosmology. This gives a reasonable fit to the data. Other models are discussed by Cohen et al. (in preparation).

Assume that $z$ measures distance in a Friedmann universe, and that we are dealing with thin randomly-oriented jets containing luminous blobs all moving with the same $\gamma$. Then the observed proper motions will have an upper envelope given by

$$
\mu \approx 2.11 \times 10^{-4} \gamma H_{0} g\left(q_{0}, z\right) \operatorname{mas} \mathrm{yr}^{-1},
$$

for $\gamma^{2} \gg 1$, where $g\left(q_{0}, z\right)$ is given by

$$
g\left(q_{0}, z\right)=q_{0}^{2}(1+z)\left(q_{0} z+\left(q_{0}-1\right)\left(\sqrt{1+2 q_{0} z}-1\right)\right)^{-1} .
$$

The solid line shows this envelope for $\gamma H_{0}=900 \mathrm{~km} \mathrm{~s}^{-1} \mathrm{Mpc}^{-1}$, and $q_{0}=0.5$. This curve could reasonably well form an upper limit to the data. The two high points (CTA 102 and 3C 395) need confirmation, but if taken at face value, they would require $\gamma H_{0} \sim 1800$.

In a randomly oriented sample of objects, the distribution of points below the upper envelope is predicted by the model; e.g., half the sources lie closer to the line of sight than $\theta=60^{\circ}$. The dashed line shows this median $\mu(z)$ for the particular case $\gamma=4$ and $H_{0}=100 \mathrm{~km} \mathrm{~s}^{-1} \mathrm{Mpc}^{-1}, q_{0}=0.5$. The position of the median line does not change much if $\gamma>4$, while for a smaller Hubble constant, it moves down. Thus we would expect 


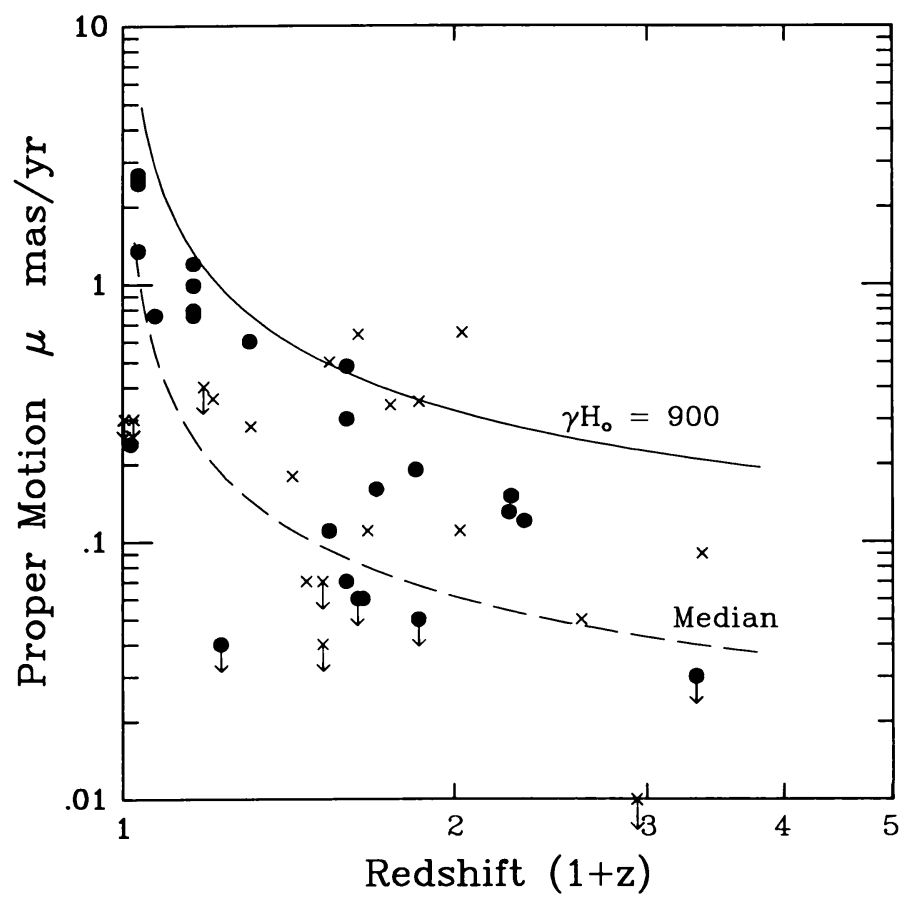

Figure 1 The $\mu-z$ diagram. The filled circles and crosses show observed sources; the solid line is an upper bound in Friedmann cosmology for a particular value of $\gamma$ and $H_{0}$; the dashed line shows the median proper motion in an orientation-unbiased sample.

less than half of the points to lie above the dashed line. This is obviously not the case, and we conclude that the sources in the Figure must be predominantly aligned in our direction.

An earlier version of this work was published by Cohen 1987, in Superluminal Radio Sources, ed. Zensus and Pearson (Cambridge: Cambridge University Press). 